\title{
Grain Boundary Decohesion by Sulfur Segregation in Ferromagnetic Iron and Nickel -A First-Principles Study-
}

\author{
Masatake Yamaguchi, Motoyuki Shiga and Hideo Kaburaki \\ Center for Computational Science and e-Systems (CCSE), Japan Atomic Energy Agency (JAEA), \\ Tokai-mura, Ibaraki 319-1195, Japan
}

Using first-principles calculations, we simulate grain boundary decohesion (embrittlement) in ferromagnetic bcc $\mathrm{Fe} \Sigma 3(111)[1 \overline{1} 0]$ and fcc $\mathrm{Ni} \Sigma 5(012)[100]$ symmetrical tilt grain boundaries by progressively adding sulfur atoms to the boundaries. We calculate the segregation energy of sulfur atom, tensile strength, and cohesive energy of the grain boundaries. We show that a certain amount of sulfur segregation (two atomic layers, 14.4 atom $/ \mathrm{nm}^{2}$ ) is energetically possible to realize considering the calculated segregation energies. At this concentration, the tensile strength and the cohesive energy of the grain boundaries reduce by one order of magnitude comparing with no segregation case. [doi:10.2320/matertrans.47.2682]

(Received May 22, 2006; Accepted August 9, 2006; Published November 15, 2006)

Keywords: grain boundary, embrittlement, first-principles calculation, sulfur, iron, nickel

\section{Introduction}

It is well known that a small amount of impurity (solute) elements bring about a drastic change on the mechanical properties of metals. For example, sulfur segregates to grain boundaries (GBs) of metals and makes metals brittle. However, the microscopic mechanism of this grain boundary decohesion (embrittlement) has been not known in detail.

Recently, we have modeled the grain boundary decohesion (embrittlement) by progressively adding sulfur atoms to a nickel grain boundary. ${ }^{1-3)}$ Our first-principles calculations have revealed that the weakening of the boundary is caused by the aggregation of sulfur atoms at the boundary, which repel each other. Because the nickel-sulfur bonds are stronger than the sulfur-sulfur bonds, the sulfur atoms are forced into non-ideal bonding and therefore push apart each other when they are neighboring.

In this paper, we show a refined result for the Ni-S system and add a new result for the Fe-S system. The improvement points in the method of calculations are as follows; (i) spinpolarized calculations are performed, (ii) unit cell including vacuum region is used; this cell allows the grain boundary sliding, (iii) the segregation energies for larger number of segregation configurations of sulfur atoms are calculated, (iv) structure relaxation is introduced into tensile test calculations, and the cohesive energy of grain boundary is also shown with tensile strength. Although the results for fcc $\mathrm{Ni} \Sigma 5$ GB do not change significantly from our previous results, ${ }^{1-3)}$ the plausibility of these results are more firmly confirmed by the above improvements.

\section{Method of Calculation}

\subsection{Electronic structure calculation}

The electronic structure calculations and crystal structure relaxations by force minimizations are performed using Vienna Ab initio Simulation Package (VASP) with Projector Augmented Wave (PAW) potentials. ${ }^{4-7)}$ The cutoff energy for the plane wave basis set is $280 \mathrm{eV}$. The Monkhorst Pack $\mathrm{k}$-point mesh is $3 \times 3 \times 1$ for both $\mathrm{Fe} \Sigma 3$ and Ni $\Sigma 5$ GBs. The
Methfessel-Paxton smearing method with $0.1-\mathrm{eV}$ width is used. All calculations are done for ferromagnetic state.

\subsection{Modeling of grain boundary}

It is known that the grain boundary embrittlement is caused by the weakening of large angle (more than $15^{\circ}$ ) grain boundaries. In the large angle grain boundaries, we choose bcc Fe $\Sigma 3(111)[1 \overline{1} 0]$ and fcc Ni $\Sigma 5(012)[100]$ symmetrical tilt grain boundaries (STGB) for the following reasons.

Table 1 shows some characters of the GBs. Although there is a difference in crystal structure (bcc vs. fcc), tilt angles of these two GBs are large and similar with each other. In addition, the atomic areal density of atoms in each atomic plane parallel to the GB plane is almost the same for the two GBs (7.2 atom $\left./ \mathrm{nm}^{2}\right)$.

Figure 1 shows the GB structures. The atomic sites are labeled by numbers and characters. The site 0 indicates grain boundary vacancy site, in which $\mathrm{S}$ atom can enter. The other sites are Fe sites in Figs. 1(a)-(b) or Ni sites in Figs. 1(c)-(d), at which $\mathrm{S}$ atom can be substituted for $\mathrm{Fe}$ or $\mathrm{Ni}$ atom.

It is well known that the disordering of atomic positions at the GB almost disappears in a few atomic layers (about $0.5 \mathrm{~nm}$ ). For this reason, the unit cells shown in Fig. 1 have sufficient length $(2.55 \mathrm{~nm})$ in c-axis direction. As for the lateral direction (in ab plane), these cells have $4(2 \times 2)$ metal atoms $\left(7.2\right.$ atom $\left./ \mathrm{nm}^{2}\right)$ in each atomic plane, where atoms are on crystallographic equivalent sites.

These unit cells have vacuum regions. The distance between the two surfaces (upper and lower surfaces) that sandwich the vacuum region is about $1 \mathrm{~nm}$, which is so long

Table 1 Grain boundary characters (hkl) indicates the crystallographic plane parallel to the GB, and [hkl] the tilt axis. The area of (hkl) plane in the unit cell is shown.

\begin{tabular}{lcc}
\hline & bcc Fe $\Sigma 3(111)[1 \overline{1} 0]$ & fcc Ni $\Sigma 5(012)[100]$ \\
\hline tilt angle & $70.5^{\circ}$ & $53.1^{\circ}$ \\
Area of (hkl) plane & $0.556 \mathrm{~nm}^{2}$ & $0.554 \mathrm{~nm}^{2}$ \\
atomic density & 7.2 atom $/ \mathrm{nm}^{2}$ & 7.2 atom $/ \mathrm{nm}^{2}$ \\
\hline
\end{tabular}


(a)

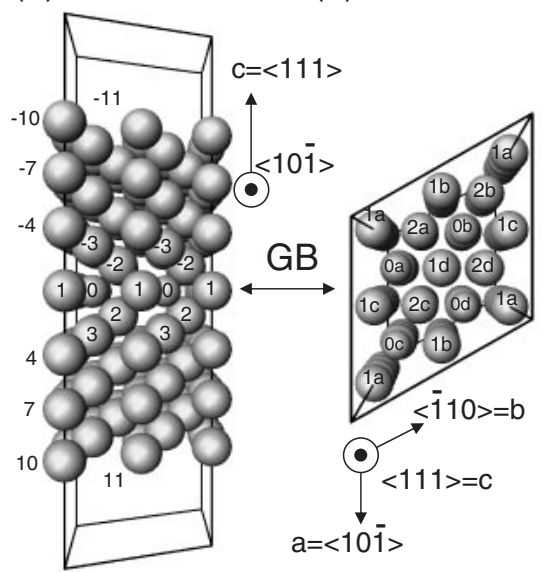

(c)

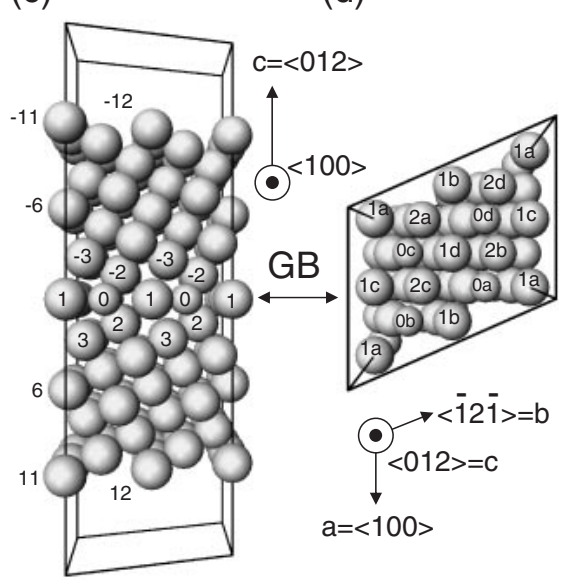

Fig. 1 Unit cell modeling of bcc Fe $\Sigma 3(111)[1 \overline{1} 0]$ and fcc Ni $\Sigma 5(012)[100]$ symmetrical tilt grain boundary (STGB). Unit cell shapes are shown by solid lines. Atomic sites (layers) are indicated by numbers $(-2,0,1,2$, etc.), in which the lateral atomic sites are indicated by characters (a, b, c, d). The radius of sphere except for site 0 is $0.1 \mathrm{~nm}$. (a) Side view of the bcc Fe $\Sigma 3$ STGB unit cell. (b) Top view of the fractured free surface at the bcc Fe $\Sigma 3$ STGB. (c) Side view of the fcc Ni $\Sigma 5$ STGB unit cell. (d) Top view of the fractured free surface at the fcc Ni $\Sigma 5$ STGB.

(a)

(b)

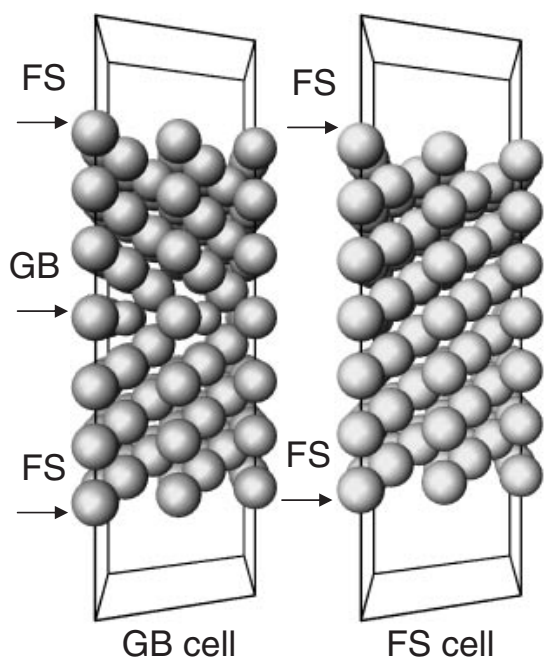

(c)

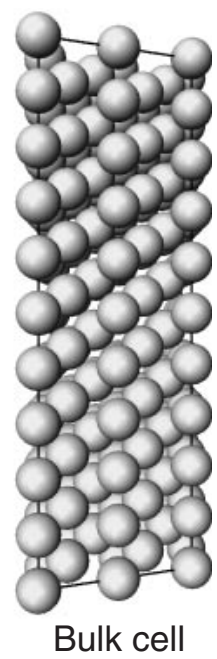

Fig. 2 (a) This is the same as Fig. 1(a). We refer this cell as "GB cell". (b) This cell does not have grain boundary (GB) but has two free surfaces (FSs) and the same number (76) of Fe atoms in similar to the GB cell. We refer this cell as "FS cell". (c) This cell has the same a and b-axis lengths of GB, FS cells. The c-axis length of this "Bulk cell" is $2.45(2.36) \mathrm{nm}$ for $\mathrm{Fe}(\mathrm{Ni})$ system, which is slightly shorter than that $(2.55 \mathrm{~nm})$ of $\mathrm{GB}, \mathrm{FS}$ cells to include bulk crystals of $120 \mathrm{Fe}(\mathrm{Ni})$ atoms.

that the interaction between the two surfaces is negligible. This vacuum region is introduced to allow GB sliding; the upper half metal block can move freely parallel to the GB plane with respect to the lower half block.

The following explanation in Ni case holds also in Fe case. From now on, we refer this unit cell as "GB cell". On the other hand, we also use a different cell that does not include grain boundary, which is referred as "FS cell" as shown in Fig. 2(b). The total energy difference between the two cells corresponds to grain boundary energy. The total energy of bulk system per one metal atom, $E_{\text {tot,atom }}^{\text {Bulk }, \text { is calculated using }}$ Bulk cell (120 atom/u.c.) as shown in Fig. 2(c). We

confirmed that this energy is almost the same as the total energy of one metal atom calculated using a primitive unit cell ( 1 atom/u.c.). The free surface energy is obtained by subtracting $E_{\text {tot, atom }}^{\text {Bulk } N i} \times N_{N i}^{F S, 0}\left(N_{N i}^{F S, 0}\right.$ : number of Ni atoms in the FS cell) from the total energy of the FS cell.

\subsection{Calculations of binding energies}

Using these unit cells, we calculate the binding energy of segregated S atoms ( $E_{b}^{G B}$ (config.)) depending on segregated sulfur configuration in the following way.

$$
\begin{aligned}
E_{b}^{G B}(\text { config. })= & E_{\text {tot }}^{G B}\left(N_{N i}, N_{S}\right)-N_{s} E_{\text {tot }}^{\text {atom } S} \\
& -E_{\text {tot }}^{G B}\left(N_{N i}^{G B, 0}, 0\right)-\left(N_{N i}-N_{N i}^{G B, 0}\right) E_{\text {tot }, \text { atom }}^{B u l, N i}
\end{aligned}
$$

Here, this binding energy is calculated with respect to the total energy of an isolated sulfur atom in vacuum, $E_{\text {tot }}^{\text {atom },}$, and also to that of metal (Ni) bulk crystal per one atom, $E_{\text {tot } \text { atom }}^{\text {But } N i}$. $E_{t o t}^{G B}\left(N_{N i}, N_{S}\right)$ is the calculated total energy for the relaxed structure of the system that includes $N_{N i} \mathrm{Ni}$ atoms and $N_{S} \mathrm{~S}$ atoms using the GB unit cell. $E_{\text {tot }}^{\text {atom } S}$ is the calculated total energy of a $S$ atom that exists in vacuum $(1 \times 1 \times 1$ in $\mathrm{nm})$. $N_{N i}^{G B, 0}$ is the number of $\mathrm{Ni}$ atoms without $\mathrm{S}$ segregation in the GB cell.

The binding energy of one $\mathrm{S}$ atom in fcc bulk Ni crystal is calculated as follows.

$$
\begin{aligned}
E_{b}^{\text {Bulk }}= & E_{\text {tot }}^{F S}\left(N_{N i}^{F S, 0}-1,1\right) \\
& -E_{\text {tot }}^{a t o m}-E_{\text {tot }}^{F S}\left(N_{N i}^{F S, 0}, 0\right)+E_{\text {tot, atom }}^{\text {Bulk,Ni }}
\end{aligned}
$$

Here, $E_{t o t}^{F S}\left(N_{N i}^{F S, 0}-1,1\right)$ is calculated when one $\mathrm{S}$ atom is substituted for one Ni atom near the center of the FS cell. This corresponds to the situation that one $S$ atom exists in inner bulk region, and thereby give an energy of solution.

Using the above energies, the total segregation energy for $\mathrm{S}$ atoms is calculated as follows.

$$
E_{\text {seg }}^{\text {total }}(\text { config. })=E_{b}^{G B}(\text { config. })-N_{s} E_{b}^{\text {Bulk }}
$$




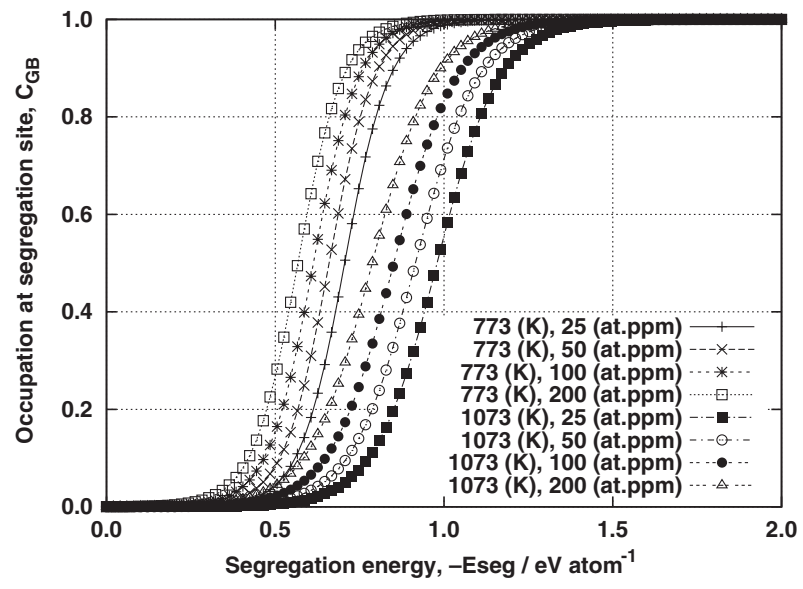

Fig. 3 McLean's curves. For each curve, ageing temperature $\mathrm{T}(\mathrm{K})$ and bulk impurity concentration $\mathrm{C}_{\text {bulk }}$ (atomic perts per million, at.ppm) are shown.

This energy indicates how much energy is obtained by moving $\mathrm{S}$ atoms from inner bulk region to the GB region. The average segregation energy per one sulfur atom, $E_{\text {seg }}^{a v}$ (config.) is obtained to divide $E_{\text {seg }}^{\text {total }}$ (config.) by $N_{s}$. Since the total energy of the system is negative, the larger the negative segregation energy, the more $\mathrm{S}$ atom can segregate.

Figure 3 shows some plots of McLean's equation ${ }^{8)}$ for some segregation conditions (ageing temperature and bulk impurity concentration). McLean's equation is based on a simple statistical model to predict segregation concentration after the system reaches thermal equilibrium. McLean derived the temperature dependence of grain boundary segregation to be,

$$
C_{G B}=\frac{C_{b u l k} \exp \left(E_{\text {seg }} / R T\right)}{1+C_{\text {bulk }} \exp \left(E_{\text {seg }} / R T\right)},
$$

where $C_{G B}$ is the grain boundary segregation concentration (occupation: 0-1), $C_{\text {bulk }}$ the bulk impurity concentration $(0-1), E_{\text {seg }}$ the segregation energy at segregation site in grain boundary region. Here, the concentration dependence of $E_{\text {seg }}$ is not taken into account.

From Fig. 3, we can understand that the value of -1.0 $\mathrm{eV} /$ atom is so large segregation energy that the segregation concentration (occupation) can be more than $0.5(50 \%)$ even for low bulk concentration (25 at.ppm) and high ageing temperature $(1073 \mathrm{~K})$.

\subsection{Tensile test calculations}

Here, we show how to calculate the cohesive energy $(2 \gamma)$ and the maximum tensile stress ( $\sigma_{\max }$, tensile strength). In our previous paper, ${ }^{1-3)}$ we performed a rigid-type tensile test calculation, where we set a fracture plane and then the upper and lower half crystal blocks are gradually separated without structure relaxations. This calculation has a large numerical error especially for high segregation concentration cases, since the sulfur atoms can be much more stable on the fractured free surfaces than in the GB as stated later. In this work, we introduce the structure relaxation in the tensile test calculation.

Ideally, the tensile test calculations should be done by

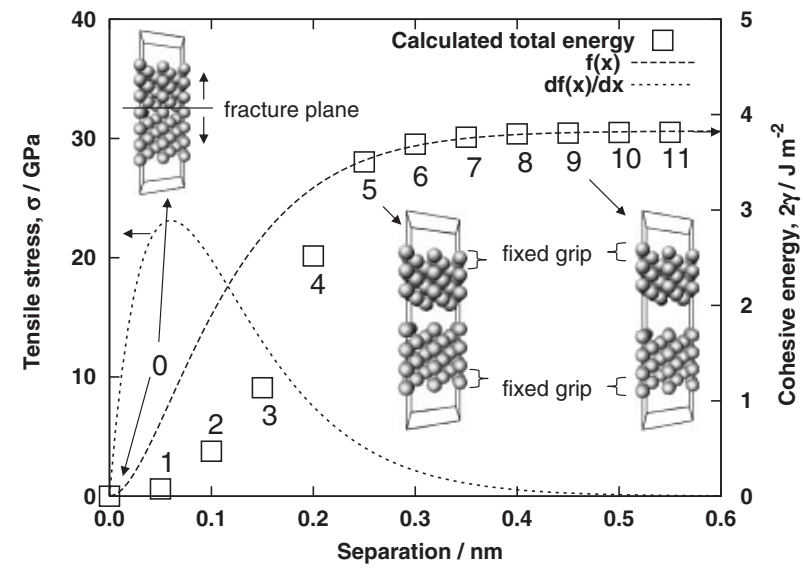

Fig. 4 Method of fitting in tensile test calculations. This is an example for clean bcc Fe $\Sigma 3$ GB.

repeating the following process: adding a small strain to the system and subsequently making structure relaxations and optimizing lateral lattice parameters (considering Poisson's ratio). However, this ideal method is very time-consuming. Furthermore, the structure relaxation becomes difficult to converge when the system has a large strain. For these reasons, we take an approximate method as stated below.

Figure 4 shows an example of tensile test calculations for clean bcc Fe $\Sigma 3$ GB. We take the following steps.

(i) A fracture plane parallel to the GB plane is assumed (point 0).

(ii) The upper and lower crystal blocks at the fracture plane are rigidly separated by some distances $(0.05 \mathrm{~nm}, 0.1 \mathrm{~nm}$, $0.15 \mathrm{~nm}, \ldots 0.5 \mathrm{~nm}$, etc.). About 10 sampling points that have different separation distances are prepared.

(iii) For each sampling point, structure relaxation is performed. Here, a few atomic layers close to the free surface are fixed to keep the two crystal blocks separated. This is usually called "fixed grip" method as shown in Fig. 4. After the structure relaxation, about 10 total energies depending on the separation distances are obtained.

(iv) The cohesive energy $(2 \gamma)$ is easily obtained from the difference between the two total energies; one is the energy of the GB without separation (point 0 ), and the other is the energy when the separation distance is so large that the energy does not change any more (point 10 or 11 ).

(v) The maximum tensile stress is obtained as follows. A simple function,

$$
f(x)=2 \gamma-2 \gamma\left(1+\frac{x}{\lambda}\right) \exp \left(-\frac{x}{\lambda}\right),
$$

is fitted to the calculated total energies. This function is well known as a universal binding curve, proposed by Rose et al., ${ }^{9}$ that describes the bonding nature between atoms well. When fitting, we ignore some total energy points where the separation distance is small (point 1-4 in Fig. 4). In this small separation region, the total energies depend on the lateral lattice parameters ( $a, b)$ due to Poisson's ratio, and on how many atomic layers are relaxed near the fracture plane. In addition, it is difficult to converge because strain energy is stored. For these difficulties, we do not use point 1-4 and instead use point 5-11 for fitting. In this large separation 
Table 2 Calculated GB energy, and free surface (FS) energy that appears after the GB is fractured.

\begin{tabular}{ccc}
\hline & bcc Fe $\Sigma 3(111)[1 \overline{1} 0]$ & fcc Ni $\Sigma 5(012)[100]$ \\
\hline GB energy & $1.48 \mathrm{~J} / \mathrm{m}^{2}$ & $1.33 \mathrm{~J} / \mathrm{m}^{2}$ \\
FS energy & $2.69 \mathrm{~J} / \mathrm{m}^{2}$ & $2.29 \mathrm{~J} / \mathrm{m}^{2}$ \\
\hline
\end{tabular}

Table 3 Calculated total magnetic moment.

\begin{tabular}{lcc}
\hline & bcc Fe $\Sigma 3(111)[1 \overline{1} 0]$ & fcc Ni $\Sigma 5(012)[100]$ \\
\hline GB cell & $180.1 \mu_{\mathrm{B}} / 76 \mathrm{Fe}$ & $55.2 \mu_{\mathrm{B}} / 84 \mathrm{Ni}$ \\
FS cell & $175.2 \mu_{\mathrm{B}} / 76 \mathrm{Fe}$ & $54.5 \mu_{\mathrm{B}} / 84 \mathrm{Ni}$ \\
Bulk cell & $257.5 \mu_{\mathrm{B}} / 120 \mathrm{Fe}$ & $73.0 \mu_{\mathrm{B}} / 120 \mathrm{Ni}$ \\
\hline
\end{tabular}

region, it is considered that Poisson's ratio does not affect significantly. Since the second derivative at $x=\lambda$ is equal to zero $\left(f^{\prime \prime}(\lambda)=0\right)$, the maximum tensile stress, $\sigma_{\max }$, is equal to

$$
f^{\prime}(\lambda)=\frac{2 \gamma}{\lambda} \exp (-1)
$$

This tensile strength, $\sigma_{\max }$, is not an accurate but an approximate value. However, it is convenient and fast way to obtain $\sigma_{\max }$, because this method avoids difficult calculations in largely strained system and can calculate all points independently. However, it is necessary to confirm that the fracture plane that is set at the beginning of the calculation is reasonable; a few different fracture planes should be tested to obtain the minimum cohesive energy and tensile strength.

\section{Results and Discussion}

\subsection{Grain boundary energy, free surface energy, and magnetic moment}

Table 2 shows grain boundary (GB) energy and free surface (FS) energy. It is well known that grain boundary energy is about $2 / 3-1 / 2$ of free surface energy. The grain boundary energies for bcc Fe $\Sigma 3$ and fcc Ni $\Sigma 5$ GBs are about $2 / 3-1 / 2$ of these free surface energies, which is in agreement with the well-known trend.

Table 3 shows the total magnetic moments for three cells as shown in Fig. 2. From this table, the average magnetic moment per $\mathrm{Fe}(\mathrm{Ni})$ atom for bulk cell is $2.15(0.61) \mu_{\mathrm{B}} /$ atom, which is in good agreement with experimental results (Fe: $2.2 \mu_{\mathrm{B}}$ /atom, Ni: $0.6 \mu_{\mathrm{B}} /$ atom).

\subsection{Segregation energy}

First, we show the segregation energy of one sulfur atom. Figure 5 shows the calculated segregation energies when one $\mathrm{S}$ atom is introduced into the GB vacancy sites (site 0 ) or substituted for a Fe/Ni atom at various sites (site 1-11 or 12). We can find the following things common to the $\mathrm{Fe} \Sigma 3$ and $\mathrm{Ni} \Sigma 5$ cases.

In the GB region, the site 2 is the most favorable segregation site and the site 0 is the second one. The site 1 and 3 are not so favorable comparing with the site 2 and 0 . The GB segregation energies at site 2 and 0 for both Fe and $\mathrm{Ni}$ cases are larger than $-1.0 \mathrm{eV} /$ atom, which is very (a) Fe $\Sigma 3$

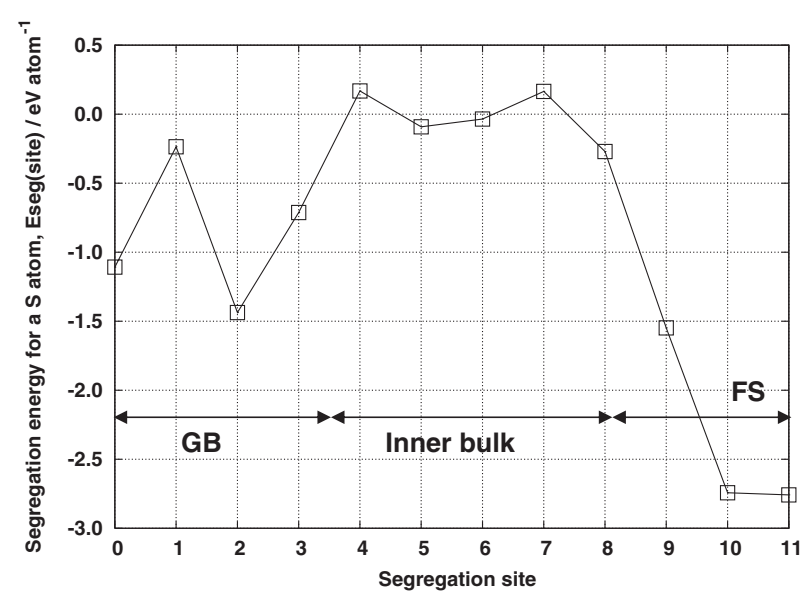

(b) $\mathrm{Ni} \Sigma 5$

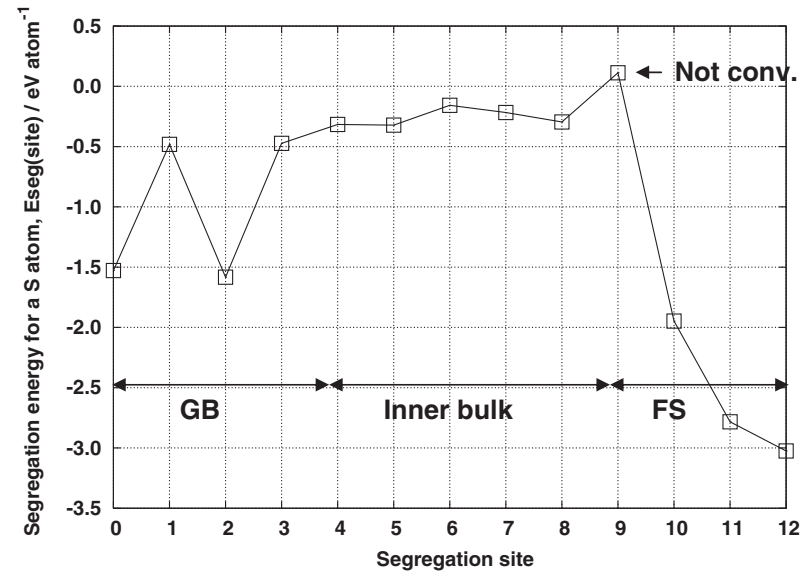

Fig. 5 Calculated segregation energies for a $\mathrm{S}$ atom in (a) bcc Fe $\Sigma 3$ GB and (b) fcc Ni $\Sigma 5 \mathrm{~GB}$. Roughly speaking, the $0-3$ sites are in grain boundary (GB) region, the 4-8,9 sites in inner bulk region, and the 8,911,12 sites in free surface (FS) region. The energy at site 9 in (b) is not a converged result.

(negative) large value as can be seen from Fig. 3. Note that the same argument holds for site $0,1,-2$, and -3 , because the grain boundary we use is a symmetrical one; there is a mirror plane at the grain boundary plane.

As can be seen from Fig. 1, site 2 and site -2 make a dumbbell pair, because the distance between the two sites is very close (about $0.25 \mathrm{~nm}$ ). This indicates that the environment (structure) around site 2 is very different from the inner bulk site. This is the reason why the segregation energy of the site 2 is very large.

In the free surface (FS) region, the outermost site (site 11 for $\mathrm{Fe} \Sigma 3$ and site 12 for $\mathrm{Ni} \Sigma 5$ ) is the most stable site for $\mathrm{S}$ atom. Its surface segregation energy is negative and larger (more stable) by $1.3-1.5 \mathrm{eV} /$ atom than any GB segregation energies. This energy difference between the GB and surface segregation energies is known as embrittling potency energy in the Rice-Wang model, ${ }^{10,11)}$ which energy indicates the extent of decohesion (embrittlement) induced by the impurity (solute).

From these results we can understand that sulfur favors grain boundary rather than inner bulk, and furthermore favors 

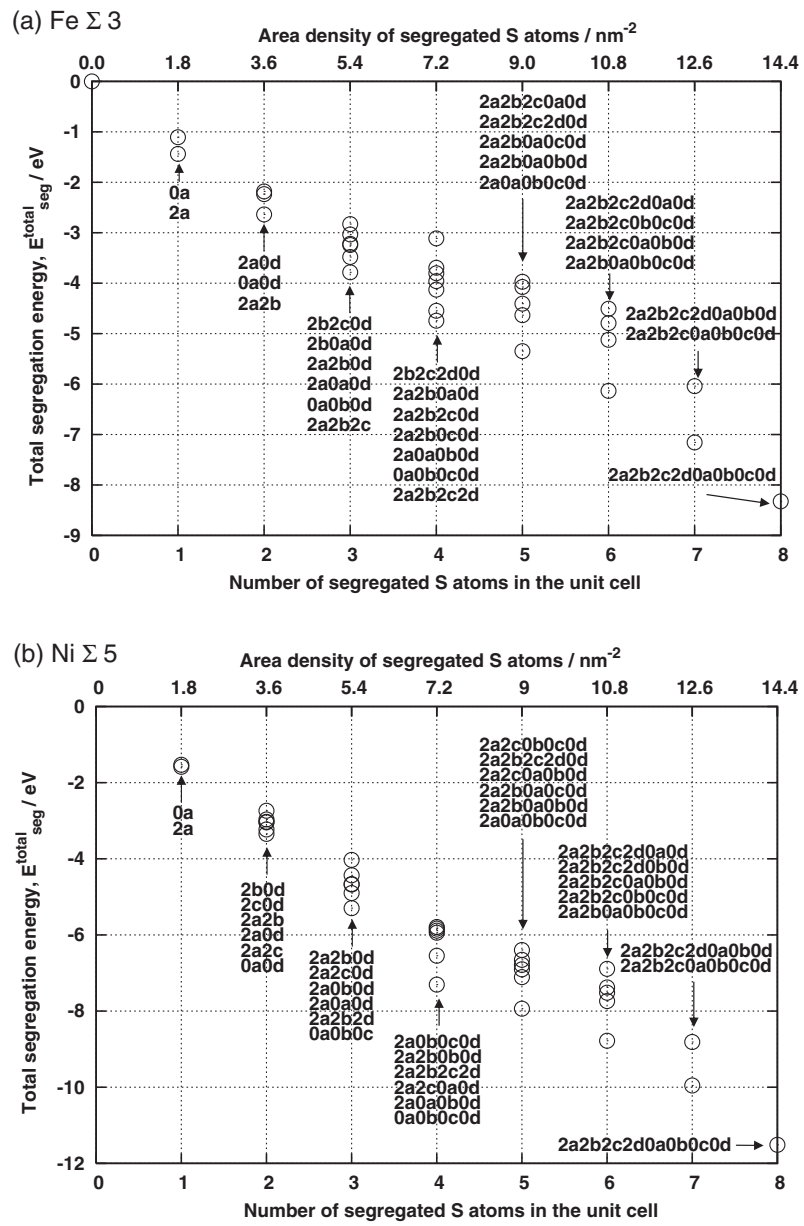

Fig. 6 Calculated total segregation energies for segregated $\mathrm{S}$ atoms in (a) bcc Fe $\Sigma 3$ GB and (b) fcc Ni $\Sigma 5$ GB. The characters ( $2 a, 0 b$, etc.) indicate the atomic site configurations for segregated $\mathrm{S}$ atoms as shown in Fig. 1. For example, the lowest energy configuration at $4 \mathrm{~S}$ atoms in (a) is " $2 \mathrm{a} 2 \mathrm{~b} 2 \mathrm{c} 2 \mathrm{~d}$ ".

surface rather than grain boundary. This trend is important for grain boundary weakening as stated later.

Next, we show the segregation energy when two or more sulfur atoms segregate to the grain boundary. Figure 6 shows total segregation energy of the system, which indicates that how much energy the system obtains when sulfur atoms move from inner bulk region to grain boundary region. Owing to the restriction of computer resources, we take only two sites ( 0 and 2 sites) for the sulfur segregation.

From Fig. 6, we can understand that the largest (negative) total segregation energy for each concentration (number of sulfur atom) increases monotonically with increasing concentration. Using the atomic site notations as shown in Fig. 1, the most stable configurations for $\mathrm{Fe} \Sigma 3$ case are 2a, 2a2b, 2a2b2c, 2a2b2c2d, 2a0a0b0c0d, 2a2b0a0b0c0d, $2 \mathrm{a} 2 \mathrm{~b} 2 \mathrm{c} 0 \mathrm{a} 0 \mathrm{~b} 0 \mathrm{c} 0 \mathrm{~d}$, and $2 \mathrm{a} 2 \mathrm{~b} 2 \mathrm{c} 2 \mathrm{~d} 0 \mathrm{a} 0 \mathrm{~b} 0 \mathrm{c} 0 \mathrm{~d}$. For Ni $\Sigma 5$ case, the most stable configurations are $2 \mathrm{a}, 0 \mathrm{a} 0 \mathrm{~b}, 0 \mathrm{a} 0 \mathrm{~b} 0 \mathrm{c}$, 0a0b0c0d, 2a0a0b0c0d, 2a2b0a0b0c0d, 2a2b2c0a0b0c0d, and $2 \mathrm{a} 2 \mathrm{~b} 2 \mathrm{c} 2 \mathrm{~d} 0 \mathrm{a} 0 \mathrm{~b} 0 \mathrm{c} 0 \mathrm{~d}$. These results can be partly understood from the fact that the distance between the crystallographic equivalent sites (the $0-0$ and 2-2 distance) is $0.4 \mathrm{~nm}$ for $\mathrm{Fe} \Sigma 3$ and $0.352 \mathrm{~nm}$ for $\mathrm{Ni} \Sigma 5$, which are much longer than the $0-2$ distance. For $\mathrm{Fe} \Sigma 3(\mathrm{Ni} \Sigma 5)$, the nearest neighbor $0-2$ distance is $0.256(0.223) \mathrm{nm}$ in no segregation case. Since
Table 4 Total segregation energy. The one and two atomic layers' cases are plotted in Fig. 6.

\begin{tabular}{lccc}
\hline Number of layers: site & Num. of S & Fe $\Sigma 3$ & Ni $\Sigma 5$ \\
\hline 1 atomic layer: 0 (or 2) & 4 & $-4.74 \mathrm{eV}$ & $-7.29 \mathrm{eV}$ \\
2 atomic layers: 0, 2 & 8 & $-8.32 \mathrm{eV}$ & $-11.51 \mathrm{eV}$ \\
3 atomic layers: $0,2,-2$ & 12 & $-10.27 \mathrm{eV}$ & $-11.92 \mathrm{eV}$ \\
\hline
\end{tabular}

(a) Average segregation energy

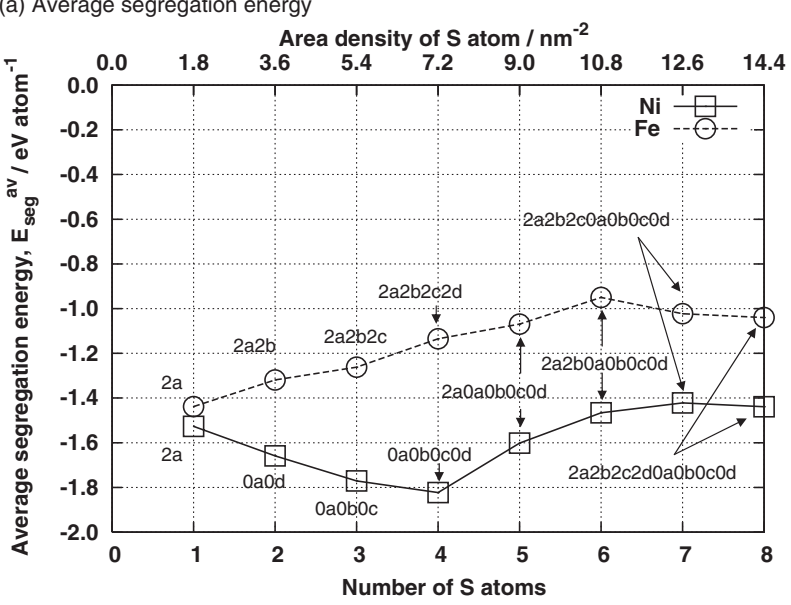

(b) Maximum tensile stress (Tensile strength)

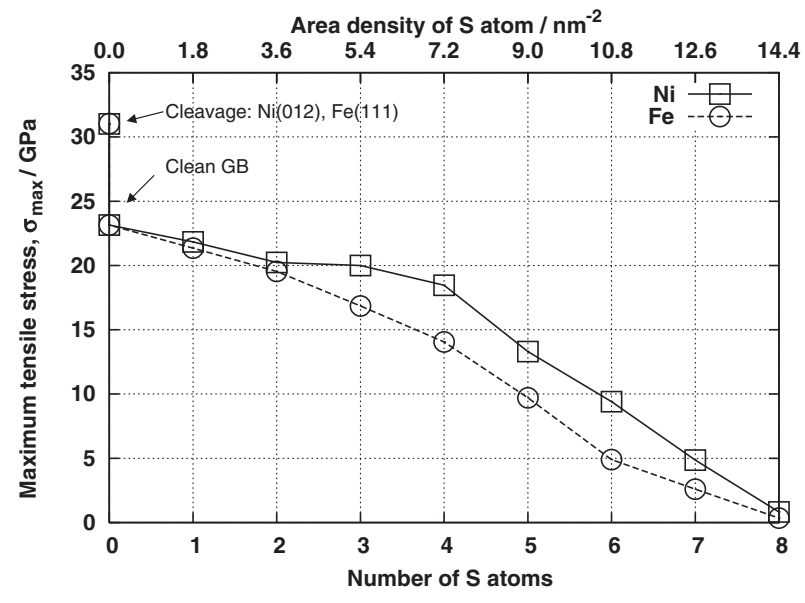

(c) Cohesive energy Area density of S atoms $/ \mathrm{nm}^{-2}$

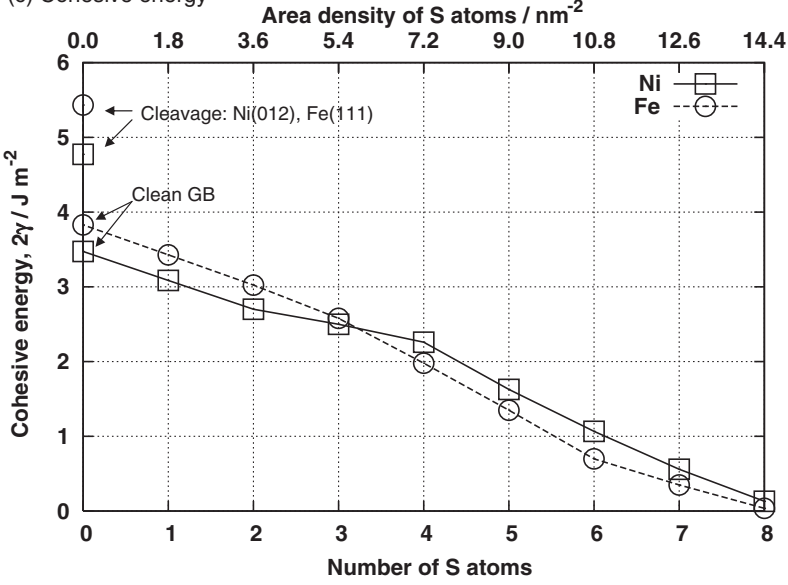

Fig. 7 (a) Calculated average segregation energies per one $\mathrm{S}$ atom in bcc $\mathrm{Fe} \Sigma 3$ and fcc Ni $\Sigma 5$ GBs. Segregated sulfur configuration is indicated by characters (0a, 2a, etc.). (b) Calculated maximum tensile stress (tensile strength). (c) Calculated cohesive energies. For (b,c), cleavage energy and tensile strength are also shown for bcc $\mathrm{Fe}(111)$ and $\mathrm{Ni}(012)$ crystal planes. 


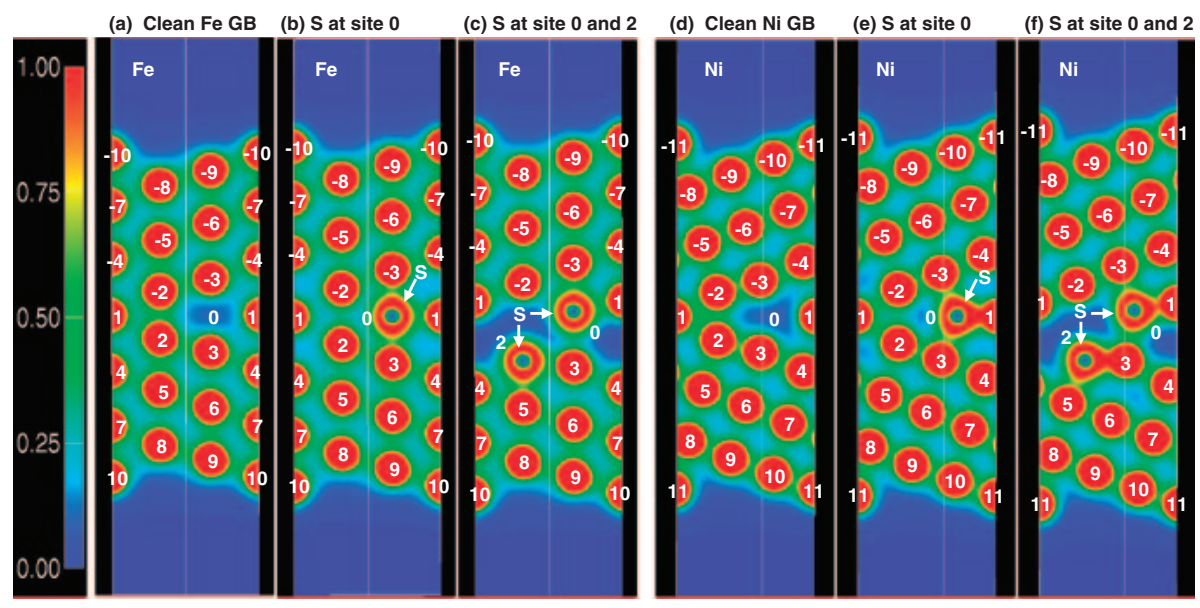

Fig. 8 Valence electron density maps in unit of electron $/(0.1 \mathrm{~nm})^{3}$ (a): Clean Fe $\Sigma 3$ GB. (b): Fe $\Sigma 3$ GB with $4 \mathrm{~S}$ segregation at site 0 . (c): Fe $\Sigma 3$ GB with $8 \mathrm{~S}$ segregation at site 0 and 2. (d) Clean Ni $\Sigma 5$ GB. (e): Ni $\Sigma 5$ GB with 4 S segregation at site 0 . (f): Ni $\Sigma 5$ GB with $8 \mathrm{~S}$ segregation at site 0 and 2 .

neighboring $\mathrm{S}$ atoms repel each other in the GB, $\mathrm{S}$ atom occupy the sites with avoiding the neighboring.

We do not consider activation energy from one configuration to the other one; we consider only total segregating energy. The final segregation configuration should be determined by the statistical method like Monte Carlo simulation. At this stage, we can at least say that the two atomic layers' segregation simultaneously at site 0 and 2 has so large segregation energy that this configuration is considered to be energetically possible to realize.

For further concentration like three atomic layers' segregation as shown in Table 4, the energy gain from segregation becomes small. In particular for $\mathrm{Ni} \Sigma 5$ case, the energy gain from two to three atomic layers' segregation is only $-0.41 \mathrm{eV}$. This indicates that the segregation tends to saturate with increasing segregation concentration.

Figures 7(a)-(c) shows the average segregation energy per one sulfur atom, calculated cohesive energy and tensile strength of grain boundary.

From Fig. 7(a), we can see that the average segregation energy increases for $\mathrm{Ni} \Sigma 5$ in negative value with increasing sulfur concentration up to one atomic layer concentration (7.2 atom $/ \mathrm{nm}^{2}$ ), whereas decreasing for $\mathrm{Fe} \Sigma 3$ case. Over 7.2 atom $/ \mathrm{nm}^{2}$, the average segregation energy decreases in negative value for both $\mathrm{Fe} \Sigma 3$ and $\mathrm{Ni} \Sigma 5$. We can not explain clearly these energy changes, because these changes depend on a small change of metal atoms' positions and the environment around the segregated $\mathrm{S}$ atoms. The most important point of these results is that these average segregation energies for all concentration are (negative) larger than about $-1.0 \mathrm{eV} /$ atom, which is considered to be very large segregation energy as can be seen from Fig. 3 (McLean's curves).

\subsection{Tensile strength and cohesive energy}

From Fig. 7(b), we can see that the tensile strengths for both $\mathrm{Fe} \Sigma 3$ and $\mathrm{Ni} \Sigma 5$ clean GBs are almost the same and about $25 \%$ smaller than the cleavage cases for $\mathrm{Fe}(111)$ and $\mathrm{Ni}(012)$ crystal planes. With increasing sulfur concentration, the tensile strength decreases. The decrease for Ni case is slower than for Fe case up to one atomic layer segregation (7.2 atom $/ \mathrm{nm}^{2}$ ). Then, both tensile strengths for $\mathrm{Ni}$ and $\mathrm{Fe}$ cases decrease rapidly over 7.2 atom $/ \mathrm{nm}^{2}$ and become close to zero at two atomic layers' segregation $\left(14.4 \mathrm{atom} / \mathrm{nm}^{2}\right)$. This change at 7.2 atom $/ \mathrm{nm}^{2}$ is due to the fact that segregated $\mathrm{S}$ atoms begin to neighbor and repel each other in the GBs.

From Fig. 7(c), we can see that the cohesive energies for clean GBs are about $25 \%$ smaller than these cleavage cases. With increasing sulfur concentration, the cohesive energy decreases. For the two atomic layers' concentration (14.4 atom $\left./ \mathrm{nm}^{2}\right)$, the cohesive energies become close to zero for both $\mathrm{Fe}$ and $\mathrm{Ni}$ cases. Comparing with tensile strength, the change at 7.2 atom $/ \mathrm{nm}^{2}$ is not so apparent in the case of cohesive energy. As stated later, we think that this is due to compensation between the energy loss by S-S repulsion and the energy gain by surface stabilization energy of $\mathrm{S}$.

\subsection{Charge density}

Figure 8 shows the calculated electron density distributions for valence electrons. Figure 8 shows clean GB case $(\mathrm{a}, \mathrm{d})$, one atomic layer's sulfur segregation case $(\mathrm{b}, \mathrm{e})$, and two atomic layers' segregation case (c,f) for bcc Fe $\Sigma 3$ and $\mathrm{Ni} \Sigma 5$ cases, respectively. We can see that sulfur makes strong covalent bonds with $\mathrm{Fe}$ and $\mathrm{Ni}$; it seems that stronger bonds are seen for Ni case than $\mathrm{Fe}$ case. For the two atomic layers' segregation cases (c,f), neighboring sulfur atoms (at site 0 and 2) can not make bonds; even push apart each other and therefore make a wide vacuum region in the GB regions.

It seems that the neighboring $\mathrm{S}$ atoms at the GBs have a strong repulsive interaction. This interaction should increase the total energy of the system; it results in the decrease of segregation energy (i.e. segregation possibility). However, the average segregation energy does not decrease significantly after $\mathrm{S}$ atoms begin to neighbor and repel each other over one atomic layer's segregation as can be seen from Fig. 7(a). Here, we think that the repulsion between neighboring $\mathrm{S}$ atoms tends to make a fracture surface as can be seen from Fig. 8(c,f), which brings about a stabiliza- 


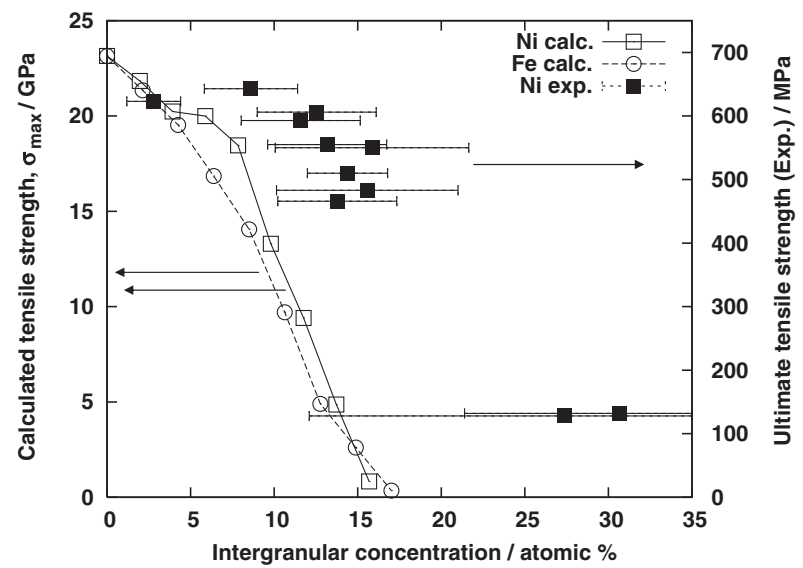

Fig. 9 Calculated tensile strength for $\mathrm{Ni} \Sigma 5$ and $\mathrm{Fe} \Sigma 3 \mathrm{GBs}$ and experimental ultimate tensile strength for fcc $\mathrm{Ni}$. Intergranular concentration indicates the concentration within $0.5 \mathrm{~nm}$ region near the GB plane in both sides. Experimental data for fcc Ni is from ref. 12

tion energy because the surface segregation energy is much larger than the GB segregation energy by about $1.2-1.5 \mathrm{eV} /$ atom as shown in Fig. 5. In other words, the enegy gain by stabilization of $\mathrm{S}$ atom on the metal surface compensates the energy loss of $\mathrm{S}-\mathrm{S}$ repulsion in the grain boundary, and therefore keeps the average segregation energy large.

\subsection{Comparison with experiments}

Figure 9 shows the comparison between calculated tensile strength and experimental ultimate tensile strength ${ }^{12)}$ of fcc $\mathrm{Ni}$. Intergranular concentration is defined as concentration within $0.5 \mathrm{~nm}$ region from the grain boundary plane in both sides. Although the order of the tensile strength is very different between calculation and experiment, both of strengths reduce by one order of magnitude with increasing sulfur concentration. The discrepancies in intergranular concentration are due to many differences between calculation and experiment; calculations are done for only one symmetrical tilt grain boundary ( $\Sigma 5$ for $\mathrm{Ni}$ ), whereas experimental fracture occurs at various kinds of grain boundaries (random grain boundaries, etc.) associated with dislocation emitting. Considering these facts, the agreement between calculations and experiments seems to be reasonable.

\section{Summary}

For ferromagnetic bcc $\mathrm{Fe} \Sigma 3(111)[1 \overline{1} 0]$ and fcc $\mathrm{Ni} \Sigma 5(012)[100]$ symmetrical tilt grain boundaries, we simulate grain boundary decohesion (embrittlement) by progressively adding sulfur atoms to the boundaries. The results for ferromagnetic $\mathrm{Ni}$ case in this work are almost the same as our previous results for non-magnetic Ni case, ${ }^{1)}$ which indicates that the spin-polarization does not affect the grain boundary decohesion significantly for $\mathrm{Ni}$ case. We found the following things in common with $\mathrm{Fe}$ and $\mathrm{Ni}$ systems.

(1) When only one sulfur atom segregate to the grain boundary region in the unit cell, the segregation energy depends on the segregation site. We found that the three sites (site 0,2 , and -2 ) are very favorable in the grain boundary region, in which the segregation energy is negative and larger than $-1.0 \mathrm{eV} /$ atom.

(2) When two or more sulfur atoms segregate, they segregate to the grain boundary with avoiding the neighboring with each other. Up to one atomic layer's segregation (4 atoms in the cell, 7.2 atom $/ \mathrm{nm}^{2}$ ), the segregated sulfur atoms tend to avoid being neighboring. This indicates that the neighboring sulfur atoms repel each other. Up to two or three atomic layers' segregation, segregated sulfur atoms neighbor and repel each other. For this reason, the energy gain by sulfur segregation gradually decreases with increasing sulfur segregation concentration; it indicates that segregation tends to saturate.

(3) The (negative) total segregation energy increases monotonically up to at least two atomic layers' segregation (14.4 atom $\left./ \mathrm{nm}^{2}\right)$. Strictly speaking, the segregation concentration should be determined by a statistical method like Monte Carlo simulation considering all segregation configurations and the activation energy between these configurations.

(4) The tensile strength and cohesive energy decreases with increasing sulfur segregation concentration. At the two atomic layers' segregation $\left(14.4\right.$ atom $\left./ \mathrm{nm}^{2}\right)$, the both of tensile strength and cohesive energy reduce to be one-tenth of the original values. This is in agreement with experimental results; the tensile strength of bulk fcc Ni finally become about one-tenth of its original strength by introducing sulfur. ${ }^{12)}$

(5) For more than one atomic layer's segregation (7.2 atom $/ \mathrm{nm}^{2}$ ), the sulfur atoms begin to neighbor each other. Over this concentration, the decreasing rate in tensile strength and cohesive energy becomes slightly larger; this trend is more apparent for Ni than Fe. This is considered to be due to a repulsive interaction between neighboring sulfur atoms.

(6) Charge density maps clearly show that the neighboring sulfur atoms in the grain boundary do not make covalent bonds with each other; even push apart each other and make a fracture surface (vacuum region) in the grain boundary. The sulfur atoms seem to be forced into non-ideal bonding because the nickel-sulfur bonds are stronger than the sulfur-sulfur bonds.

(7) Up to two atomic layers' segregation (14.4 atoms/ $\mathrm{nm}^{2}$ ), the energy loss of the neighboring S-S repulsion seems to be compensated by the energy gain from the stabilization energy of $\mathrm{S}$ atom on the metal surface, because the S-S repulsion tends to make a fracture surface in the grain boundary and the stabilization energy of $S$ atom on the metal surface is much larger than that in the grain boundary. This may be the reason why the average segregation energy does not reduce significantly by the neighboring $\mathrm{S}-\mathrm{S}$ repulsion up to at least two atomic layers' segregation.

\section{Acknowledgments}

We thank Y. Nishiyama in JAEA and J. Kameda in University of Pennsylvania for helpful discussions. 


\section{REFERENCES}

1) M. Yamaguchi, M. Shiga and H. Kaburaki: Science 307 (2005) 393 397, published online 6 January 2005.

2) M. Yamaguchi, M. Shiga and H. Kaburaki: Science 309 (2005) 1677d (response to comment)

3) W. T. Geng, J.-S. Wang and G. B. Olson: Science 309 (2005) 1677c (comment).

4) G. Kresse and J. Hafner: Phys. Rev. B 47 (1993) 558.

5) G. Kresse and J. Furthmüller: Phys. Rev. B 54 (1996) 11169.
6) G. Kresse and D. Joubert: Phys. Rev. B 59 (1999) 1758

7) P. E. Blöchl: Phys. Rev. B 50 (1994) 17953.

8) D. McLean: Grain Boundaries in Metals, (Oxford Univ. Press, London, 1957).

9) J. H. Rose, J. Ferrante and J. R. Smith: Phys. Rev. Lett. 47 (1981) 675.

10) J. R. Rice and J.-S. Wang: Mater. Sci. Eng., A 107 (1989) 23.

11) R. Wu, A. J. Freeman and G. B. Olson: Science 265 (1994) 376.

12) J. K. Heuer, P. R. Okamoto, N. Q. Ram and J. F. Stubbins: J. Nucl. Mater. 301 (2002) 129. 